Jadwiga TUDEK

Uniwersytet Warszawski

\title{
La politique de soutien au cinéma en France
}

\section{Introduction}

T e chiffre d'affaires du cinéma en France en 2002 s'est élevé à 1,027 $\smile_{\text {mld d'EUR }}{ }^{1}$ ce qui est peu, comparé à d'autres segments du marché des médias tels que la presse (10,35 mld d'EUR en 2002)² ou la télévision $(6,86 \text { mld d'EUR })^{3}$. N'étant relativement pas le médias majeur de point de vue économique, la cinématographie française n'en reste pas moins la première industrie de ce type au niveau européen ${ }^{4}$ et l'une des priorités de la politique culturelle française.

En effet, le fonctionnement du cinéma en France s'imagine mal aujourd'hui sans l'intervention permanente des pouvoirs publics. Voyant très tôt le rôle que le Septième Art joue en tant qu'élément créateur de l'identité nationale, l'Etat a voulu porter secours à un secteur qui à toujours eu du mal à se financer à cause du caractère incertain de ce genre d'investissements. D'autant plus, qu'au cours de la seconde moitié du $\mathrm{XX}^{\mathrm{e}}$ siècle, la domination de la télévision dans le secteur des médias et la concurrence des producteurs américains, ont encore affaibli cette industrie, déjà assez fragile.

Les bases du dispositif de soutien au cinéma en France, tel que nous le connaissons aujourd'hui, ont été élaborés après la Seconde Guerre mondiale avec les trois lois d'aide qui se sont succédées en 1948, 1953 et 1959. Se voulant d'abord temporaires, ces programmes de soutien ont été ensuite remaniés de manière à être compatibles avec le droit européen et le sy-

1 Statistiques annuelles 2002, Centre National de la Cinématographie (www.cnc.fr).

2 Direction du développement des médias (www.ddm.gouv.fr).

3 Conseil Supérieur de l'Audiovisuel (www.csa.fr).

4 Avec une production de 204 films en 2001, la France se place en première position parmi les producteurs de films européens, en devançant l'Espagne (106 films produits au cours de la même période) et l'Italie (103 films produits) - source: EUROSTAT (www.europa.eu.int/comm/eurostat/). 
stème a été successivement enrichis par de nouvelles dispositions et aides, englobant pratiquement tous les acteurs du secteur cinématographique. Depuis 1946, l'administration de ce secteur en France est confiée à un établissement public à caractère administratif - Le Centre National de la Cinématographie, placé sous l'autorité du Ministère de la Culture et de la Communication. Cette institution, sans équivalent sur les autres secteurs industriels, exerce une tutelle administrative, financière voire politique sur le secteur du cinéma en France, ces missions principales étant: le réglementation dans le domaine de l'industrie du cinéma, le soutien à l'économie du cinéma, de l'audiovisuel et du multimédia, le promotion du cinéma et de l'audiovisuel et leur diffusion aussi bien en France qu'a l'étranger ainsi que la protection et la diffusion du patrimoine cinématographique ${ }^{5}$. Depuis 1984 les compétences de cet organe d'Etat ont été étendues au soutien de l'industrie audiovisuelle.

Le soutien à la cinématographie qui englobe aujourd'hui pratiquement toutes les étapes de la fabrication et de la commercialisation du produit cinématographique est centré autour de deux axes majeurs. D'une part, un rôle décisif dans le développement de cette industrie est accordé au diffuseurs de programmes de télévision qui se sont vus imposer un nombre important d'obligations et de contraintes tels les quotas de production ou les quotas de l'emission, servant à stimuler la production, la distribution ainsi que l'exploitation de films en France. D'autre part le cinéma français bénéficie aujourd'hui d'un système développé d'aides aussi bien au niveau national, régional qu' européen, dont l'élément central constitue le Compte de soutien financier à l'industrie cinématographique et à l'industrie des programmes audiovisuels gérée par le CNC.

\section{a) Les obligations des chaînes de télévision}

Le financement du cinéma en France s'appuie en grande partie sur les quotas de production imposés aux diffuseurs de programmes. La production nationale est d'autre part stimulée par le système de quotas d'émission et les obligations de programmation spécifiques à chaque chaîne auxquelles s'ajoute le réglementation faisant du cinéma l'un des secteurs interdits à la publicité télévisuelle.

5 Centre National de Cinématographie (www.cnc.fr). 


\section{Quotas de production}

C'est en 1974, que l'une des chaînes hertziennes françaises (FR3) s'est vue imposer l'obligation de financement des oeuvres cinématographiques. Le principe des quotas de production qui depuis a été élargi aux autres chaînes hertziennes ainsi qu'aux chaînes du câble et du satellite a été clairement défini dans la loi du 30 septembre $1986^{6}$, mais ces obligations pour les différents types de diffuseurs ont ensuite été précisés dans des décrets successifs?

Ainsi, d'après les dispositions du décret du 17 janvier 1990, les chaînes hertziennes en clair (à part La Cinquième, qui diffuse moins de 52 films par an) sont obligées de consacrer $3 \%$ de leur chiffre d'affaires à des dépenses contribuant au développement de la production d'œuvres cinématographiques. Sur ce chiffre, $2,5 \%$ doit être consacré à la production d'œuvres d'expression originale française, les 0,5\% restant s'appliquant aux dépenses contribuant au développement de productions européennes. Le décret du 28 décembre 2001 augmente encore le taux de participation des chaînes hertziennes dans le financement de la production cinématographique le situant au niveau de 3,2\% du chiffre d'affaires de chaque chaîne en clair. La participation des chaînes au financement de la production cinématographique doit cependant se faire par le biais de filiales crées spécialement à cette fin. De plus, la somme versée ne peut excéder la moitie du coût de la production de l'œuvre et elle ne doit pas être constitué, pour plus de la moitié, de sommes investies dans la production de cette oeuvre cinématographique par les filiales de la chaîne.

Cependant, le financement de l'industrie cinématographique en France s'appuie surtout sur la chaîne hertzienne à péage - Canal Plus, crée en 1984. En effet, Canal Plus est tenu de consacrer $25 \%{ }^{8}$ de ses recettes ${ }^{9}$ hors taxe d'abonnement et de publicité quel que soit le mode de diffusion à l'acquisition de programmes cinématographiques. Les ouvres d'expres-

6 Loi $\mathrm{n}^{\circ}$ 86-1067 du 30.09.1986 modifiée par la loi du 18.01.1992.

7 Il s'agit de documments suivants: décrets $n^{\circ} 90-66$ du 17.01.1990 modifié le 27.03.1992, décret $n^{\circ} 90-67$ du 17.01.1990 modifié le 27.03.1992, décret $n^{\circ} 95-1162 \mathrm{du}$ 6.11.1995, décret du 11.03.1999 et décret du 28.12.2001.

8 Un rabais de 5\% sur cette somme est en general accordé à la chaîne annuellement pour payer les coût du codage des émissions.

9 Par ressources totales, on entend pour Canal + produit des abonnements, recettes publicitaires et parrainage. 
sion originale française ${ }^{10}$ doivent constituer $45 \%$ de cette somme, tandis que les oeuvres européennes - 15\%. En contrepartie d'une participation aussi importante dans le financement des films, la chaîne bénéficie de privilèges liés au délais de diffusion d'œuvres. En effet, Canal Plus a obtenu le droit de diffusion de films français 1 an après leur sortie en salles, alors que pour les autres chaînes hertziennes ce délai est de 3 ans ou 2 dans le cas d'une coproduction. Canal Plus, accorde, d'autre part, des primes supplémentaires à des films, achetés sur scénarios, réalisant plus de 500000 entrées en salles dans la limite de $0,5 \%$ de son chiffre d'affaires et bien qu'aucune disposition légale ne soit faite à ce sujet, elle participe à de nombreuses productions en caractère de coproducteur à travers sa filiale "Studio Canal". Finalement, la chaîne crypté tout comme les autre chaînes à péage consacrées au cinéma est tenue de verser chaque année une contribution à la distribution et à l'exploitation des films en salles.

Le poids de ces investissements fait de Canal Plus le premier partenaire économique du cinéma français. En effet, rien qu'en 2001, sa participation dans les investissements dans la production cinématographique française s'élevait à $34 \%$ soit $229 \mathrm{mln}$ d'EUR ${ }^{11}$. Un chiffre auquel il faudrait ajouter la somme servant à financer la distribution et l'exploitation d'élevant à 18,3 mln d'EUR, ainsi que de nombreux investissements de Studio Canal Plus dans la production cinématographique européenne. Ces chiffres confirment la position dominante de Canal Plus sur le marché du cinéma en France. En effet, la chaîne, appelée à juste titre „le poumon du cinéma français”, est aujourd'hui l'un des premiers facteurs de son développement et sa condition économique influence celle de tout le secteur cinématographique.

Conformément aux dispositions des décrets du 1 septembre 1992 et du 24 janvier 1995, basant en majeure partie sur les dispositions de la directive européenne, la Télévision sans Frontières du 3 octobre 1989, la production cinématographique est également financé par les autres chaînes de la télévision à péage ${ }^{12}$. Les décrets imposent des obligations spéciales, dans

10 D'après la definition du décret du 27.03.1992, sont qualifiées d'oeuvres d'expression originale française toutes productions cinématographiques et audiovisuelles réalisées integralement ou en majeure partie en français ou dans une des langues régionales parlées sur le territoire français.

11 R. Bonnel, La vingt-cinquiéme image - une économie de l'audiovisuel, Gallimard, Paris 2001, p. 414.

12 Selon les dispositions de la directive en question les diffuseurs de programmes de télévision doivent consacrer au moins $10 \%$ du budget ou $10 \%$ du temps d'antenne à des productions independantes européennes. 
ce domaine, aux chaînes thématiques du cinéma qui doivent consacrer de 20 a $21 \%$ de leur chiffre d'affaires (hors TVA) à l'achat des droits de diffusion de productions européennes, les oeuvres d'expression originale française devant constituer de 14 à $17 \%$ de ce chiffre. Le pourcentage du chiffre d'affaires de ces chaînes constituant les quotas de production augmente en fonction du nombre d'abonnés de cette chaîne.

Un régime spécial au niveau du financement de la production cinématographique concerne également la pay per view ${ }^{13}$. D'après l'art 21 du décret du 1 septembre 1992 la part minimale que se service est tenu de consacrer à l'achat de droits de diffusion d'œuvres cinématographiques est fixé par avis d'une commission constitué par arrêté du ministre chargé de la communication auprès du $\mathrm{CNC}$ et en fonction notamment du nombre de foyers considérés.

En conclusion, il faut souligner l'importance que joue aujourd'hui la télévision dans le financement de la production cinématographique en France. Il faut noter également l'évolution considérable qui c'est opérée dans la législation française pour ce qui est du principe des quotas de production. Ne concernant en 1974 qu'une seule chaîne, ce principe a été élargi graduellement à toutes les formes de diffusion (télévision hertzienne en clair et à péage, le câble et le satellite, les services de pay per view). Avec le développement de nouvelles technologies et l'évolution du paysage télévisuel français, les quotas de production trouvent de nouvelles sources d'application. Ainsi, d'après Alain Terzian, le président de l'Union française des producteurs, ce principe devrait trouver une application dans le cas des diffuseurs de productions cinématographiques sur Internet grâce à la technologie du streaming.

\section{Quotas d'émission}

La France est l'un des pays où les obligations des diffuseurs de programmes quant aux genre et provenance des émissions présentées sont les plus complexes. Certaines de ces dispositions visent à protéger les producteurs de programmes français contre la concurrence étrangère, principale-

13 Il s'agit des chaînes qui pratiquent le paiement à la séance, les services de TV étant payés par les usagers directement, en fonction de la durée de visionnage ou de l'émission visionnée. 
ment américaine. Parmi ces dispositions, il faut énumérer les quotas d'émission dont le principe a été établi dans la loi du 30 septembre 1986, qui stipule que $60 \%$ d'émissions présentées sur les chaînes françaises soient des oeuvres européennes et $40 \%$ des oeuvres d'expression originale française. Cette proportion doit être observée durant les heures de grande écoute $^{14}$ et les heures d'écoute significative, définies par le CSA pour les chaînes hertziennes privées en clair uniquement. La loi sur les quotas d'émissions en vigueur en France est l'une des plus restrictives au niveau européen et dépasse largement les obligations imposées, en cette matière, au diffuseurs par la directive européenne de $1989^{15}$. D'autre part, l'évaluation positive de ce dispositif par les pouvoirs français lui a valu d'être transposé sur d'autres secteurs du marche des médias. En effet, depuis 1996, il existe également un quotas de 40\% d'œuvres nationales à la radio française.

Les obligations de programmation

Certaines dispositions légales françaises relatives au contenu de programmes de télévision ont pour but la stimulation de la fréquentation des salles et de même le développement de la branche cinématographique. Ainsi, l'émission de films à long métrage est interdite sur les chaînes hertziennes, le mercredi ${ }^{16}$ et le vendredi soir, ainsi que le samedi pendant toute la journée et le dimanche jusqu'à 20.30. Le nombre de films à long métrage pouvant être diffusés sur une chaîne hertzienne en clair au cours de l'année ne doit pas dépasser 192, dont 104 aux heures de grande écoute (20.30-22.30). Canal Plus, la chaîne payante du cinéma et du sport, fait

14 Oeuvres audiovisuelles: tous les jours de $18 \mathrm{~h}$ à $23 \mathrm{~h}$ sauf mercredi (de $14 \mathrm{~h}$ à $23 \mathrm{~h}$ ) oeuvres cinématographiques: tous les jours de 20.30 à 22.30 .

15 La directive précise que les diffuseurs de programmes sur le territoire de l'UE doivent consacrer au moins la moitié du temps d'antenne (à l'exclusion du temps consacré aux informations, aux manifestations sportives, au jeux, à la publicité et au télétexte) à l'émission d'oeuvres européennes.Au cas où une telle proportion ne pourrait pas être atteinte, la directive recommande que le temps d'antenne consacré à l'émission d'oeuvres européennes dans un pays donné na soit pas inférieur à la moyenne enregistrée dans ce pays en 1988.

16 En France, ce jour de la semaine est traditionnellement le jour de la sortie des films en salles. 
exception à cette règle, ces obligations quant au nombre et caractère d'émission diffusées étant règlées par une convention particulière avec le $\mathrm{CSA}^{17}$.

Des dispositions précises règlent également la question de la chronologie des médias. Le délai de diffusion des oeuvres cinématographiques pour les chaînes hertziennes en clair est de 3 ans ou 2 en cas d'une coproduction. Ce délai peut être également raccourci à 18 mois sur dérogation selon accord avec les ayants-droits, conformément à l'article 7 de la directive Télévision sans frontières du 3 octobre 1989, modifié le 30 juin 1997. Pour Canal Plus, se délai n'est que d'un an.

Les secteurs interdits à la publicité télévisée

Parmi les autres facteurs, dont l'objectif est d'influencer le développement du cinéma en France, il faut citer la législation régulant le fonctionnement du marché publicitaire. En effet, d'après l'article 8 du décret $n^{\circ}$ 92-280 du 27 mars $1992^{18}$, le cinéma, tout comme le presse, l'édition, et la grande distribution, fait partie des secteurs dont la publicité à la télévision en France est interdite. Dans le cas du secteur cinématographique, cette disposition vise surtout à protéger les intérêts des moyennes et petites entreprises françaises de production, contre la concurrence de l'industrie cinématographique américaine, dont la position dominante sur le marché du cinéma en Europe se trouverait encore renforcée grâce à l'accès à la publicité télévisée. De plus, cette mesure permet aux autres médias, concurrents de la télévision d'accéder à une source additionnelle de financement.

\section{b) le Compte de soutien financier à l'industrie cinématographique et à l'industrie des programmes audiovisuels}

L'un des éléments clés du dispositif français de soutien à l'industrie cinématographique est le Compte de soutien financier à l'industrie cinéma-

17 Convention signée le 29 mai 2000.

18 Le décret du 7/10/2003 dont l'entrée en vigueur est prévue pour le $1^{\text {er }}$ janvier 2004 restreint le liste des secteurs interdits à la publicité télévisée, pour satisfaire aux exigences de Bruxelles, mais d'après la nouvelle reglementation, le cinéma restera un secteur interdit à ce genre de publicité. 
tographique et à l'industrie des programmes audiovisuels, crée en 1948 et géré par le Centre National de la Cinématographie. Depuis 1984, les aides du Compte servent également à stimuler la production audiovisuelle. Le Compte de soutien, qui a permis de préserver la cinématographie française d'une crise grave, due surtout à la concurrence d'Outre-Atlantique et à incité de nombreuses coproductions européennes est un système unique pour ce qui est du mode de son financement. En effet, ses ressources, bien qu'incluses dans le loi de finances, ne proviennent pas du budget de l'Etat mais des taxes prélevées sur les différents acteurs du marché de l'audiovisuel en garantissant ainsi une redistribution de revenus au niveau du secteur. Les ressources du Compte se composent principalement de trois taxes, prélevées sur: les sociétés de télévision, le prix des billets de cinéma ainsi que sur l'édition vidéo.

La Taxe Spéciale Additionnelle (TSA), perçue sur le prix des tickets de cinéma, assure $22 \%$ des ressources du Compte de soutien. Elle représente selon l'année de 10 à $12 \%{ }^{19}$ du prix du billet de cinéma payé par le spectateur. Les sommes perçues grâce à cette taxe servent surtout à favoriser la production et la distribution ainsi que les investissements dans le parc des sales.

La Taxe sur les ressources des sociétés de télévision, constitue aujourd'hui la ressource principale du Compte $(74,6 \%$ des ressources en 2001). C'est en 1986 que ce prélèvement de 5,5\% sur les recettes de publicité, des abonnements et du parrainage des sociétés de télévision a été inscrit pour la première fois dans la loi de finances.

La troisième ressource du Compte est la Taxe sur l'édition vidéo, crée en 1993, qui est un prélèvement de $2 \%$ sur les recettes liées à la vente et à la location des cassettes vidéo et DVD. Les sommes perçues grâce à cette taxe représentent chaque année de 2 à $3 \%$ des ressources du Compte de soutien et sont presque entièrement consacrées au secteur cinématographique.

Les autres recettes telles que: le remboursement symbolique des avances sur recettes, le prélèvement spécial sur les bénéfices des films classés $\mathrm{X}$, l'amortissement de certains prêts, l'éventuel excèdent d'exécution de l'exercice précédent, procurent moins de $1 \%$ des ressources du Compte.

D'après les estimations faites par le Ministère de la Culture et de la Communication pour l'année 2003, les ressources du Compte durant cette

19 R. Bonnel, op. cit., p. 581. 
période devraient s'élever à $449 \mathrm{mln}$ d'EUR. La somme consacrée au cinéma serait de $250,16 \mathrm{mln}$, et celle, attribuée à la production audiovisuelle de 209,13 mln $\mathrm{EUR}^{20}$.

Les aides octroyées dans le cadre du Compte de Soutien, sur la base de ces chiffres, se divisent en aides automatiques et aides sélectives.

Les aides automatiques, octroyées avant tout au producteurs de films et de programmes audiovisuels, constituent la partie centrale du système. Elles facilitent l'accumulation du capital dans la branche tout en stimulant l'activité de segments choisis du marche cinématographique, en créant une garantie financière et en assurant une protection contre la concurrence étrangère. Les aides automatiques sont octroyées à chaque producteur, distributeur ou exploitant qui remplit certains critères, du fait même de son activité commerciale. Ainsi, l'aide automatique à la production est accordée à chaque producteur qui exploite un film dit „éligible au soutien”. L'aide qu'il reçoit est une partie de la taxe perçue sur l'exploitation de ce film en salles, à la télévision et sur cassettes vidéo ou DVD, et doit être obligatoirement affectée à la production de nouveaux films à condition que d'autres dépenses privilégiées (impôts, salaires, industrie techniques etc.) ont été préalablement réglées. Les sommes perçues de cette manière doivent être obligatoirement réinvesties dans un délai de 5 ans à compter du 1er janvier de l'année suivant celle au cours de laquelle elles ont été calculées. Les aides automatiques sont également octroyées aux distributeurs et aux exploitants, servant surtout, dans le cas de ses derniers aux investissements liés à la modernisation du parc des salles.

Les aides automatiques, qui sur les 5 dernières années ont constituées $70 \%$ des dépenses du Compte de soutien, ont été l'un des éléments stimulant la production cinématographique française, mais elles n'ont pas également échappé à certaines critiques, soulignant le caractère fermé de ce genre de financement, réservé par définition à des acteurs déjà présents sur le marché, ayant une position forte dans la branche.

Pour corriger l'effet discriminatoire que peut donc avoir ce genre d'aide, les pouvoirs français ont introduit dès l'année 1959 des aides sélectives, dont le but était de donner une chance aux nouveaux talents dans l'industrie cinématographique. L',,avance sur recettes" qui est jusqu'à aujourd'hui la plus importante des aides sélectives est octroyée aux auteurs de

20 Ministère de la Culture et de la Communication, Budget 2003 (www.culture.gouv.fr). 
scénarios choisis avant la réalisation du film. Elle prend le plus souvent la forme d'un prêt sans intérêt, amortis sur les recettes du film au fur et à mesure de son exploitation en salles. Chaque année, de 30 à 50 films sont partiellement financées grâce à cette aide pour une somme de 300000 à 400000 EUR. Les critiques adressées à cette forme de soutien mettent en avant la partialité du jury dans l'octroi des aides, le caractère déficitaire de ce genre de production, la faible rentabilité des projets, et un taux presque inexistant de rendement des sommes prêtées (2-3\%) qui d'une certaine manière transforment cette aide à caractère de prêt en une subvention. De plus, de nombreux projets qualifiés pour l'octroi de l',,avance sur recettes", ont du mal à trouver des sources de financement complémentaires pour effectuer le tournage. Cependant, malgré ces arguments, l'avance sur recettes a joué un grand rôle dans le développement du cinéma français en promouvant de nouveaux talents et des projets innovants à grande valeur artistique. A côté de l'avance sur recettes, le système français de soutien au cinéma comprend également d'autre aides sélectives parmi lesquelles on peut énumérer: l'aide à l'écriture et au développement du scénario, l'aide à le réalisation de longs métrages tournés en langue étrangère, des aides aux coproductions internationales ainsi que des aides à la production de courts métrages.

Le Compte de soutien financier à l'industrie cinématographique et à l'industrie des programmes audiovisuels est aujourd'hui l'un des outils essentiels de la politique française dans le domaine des médias. En instaurant un système d'épargne forcé et de redistribution de fonds à l'intérieur du secteur audiovisuel pour financer les différents acteurs de la branche cinématographique, le Compte a été l'un des éléments majeurs qui ont permis le développement du cinéma français tout en le préservant d'une crise grave, liée à la concurrence des films américains et à la baisse de la fréquentation en salles qui a duré depuis le début des années 60 jusqu'au début des années 90. Ses aides divisées en deux catégories: aides automatiques et sélectives, destinées surtout à soutenir la production de films, englobent l'ensemble des acteurs de la branche cinématographique en France. Certes, le système compliqué de dépendances dans le cadre du Compte même pose le problème de l'effectivité de gestion des fonds et ne peut remédier aux nombreux conflits parmi les représentants de différents groupes d'intérêts. En tant que l'un des outils les plus sophistiqués de la politique française dans le domaine des médias qui se caractérise par un grand interventionnisme, il est l'objet de nombreuses critiques au niveau de l'Union Européenne et surtout de la Direction de la Concurrence. Ainsi 


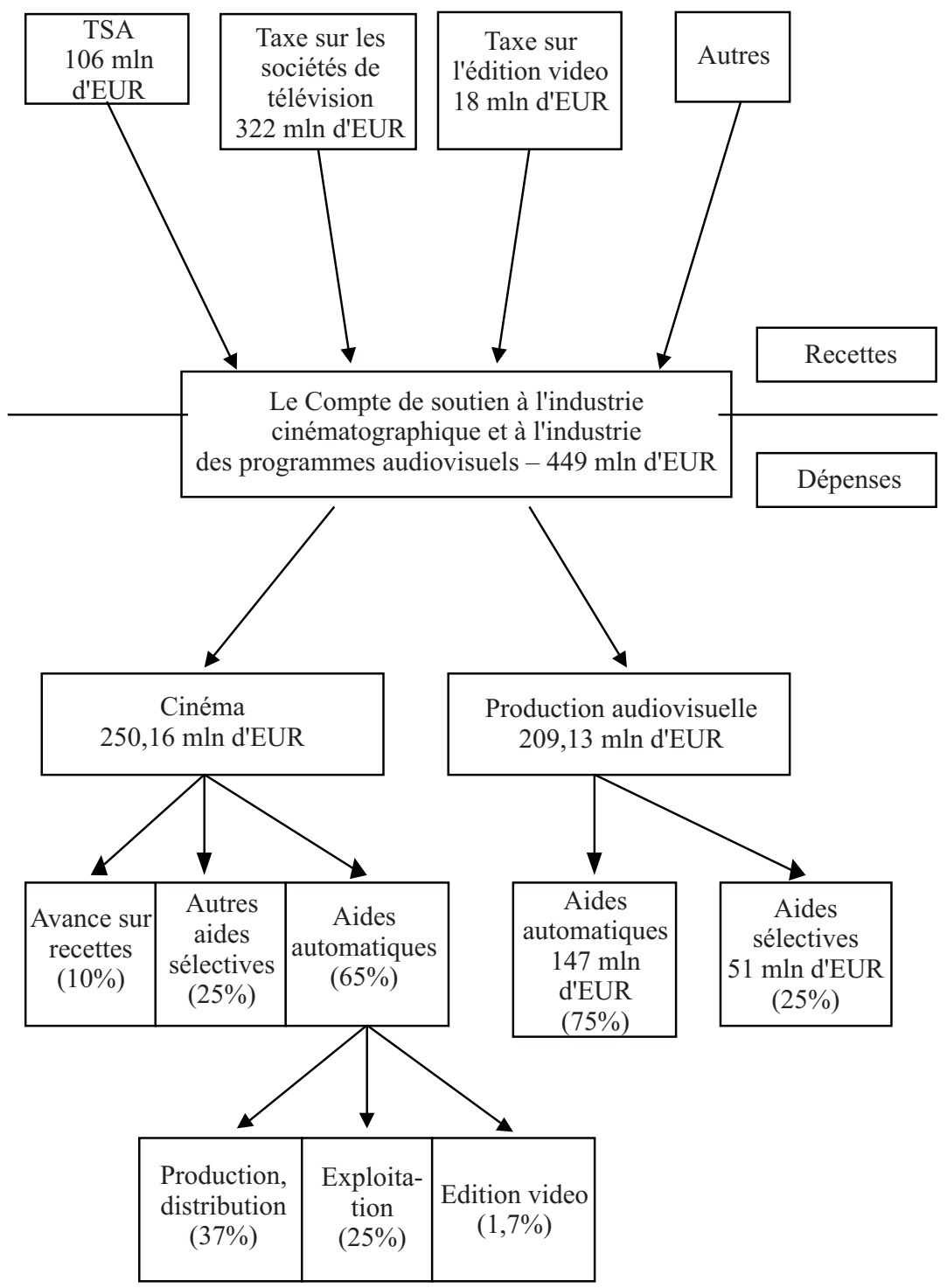

Schéma $\mathrm{n}^{0} 1$ : Le compte de soutien à l'industrie cinematographique et à l'industrie des programmes audiovisuels - estimations pour l'année 2003

Source: Centre National de la Cinématographie. 
sa survie au temps de l'intégration régionale en Europe n'est possible que grâce à de continuelles adaptations ${ }^{21}$ à l'acquis communautaire, qui compliquent d'avantage un système qui est déjà assez complexe.

Un model détaillé du fonctionnement du Compte de soutien est présenté sur le schéma $\mathrm{n}^{0} 1$.

\section{c) Le soutien fiscal}

A côté des profits découlant de l'exécution des quotas imposées aux diffuseurs ainsi que des aides distribuées par le Compte de soutien, le cinéma en France bénéficie également de différents avantages fiscaux.

Premièrement, un taux réduit de TVA $(5,5 \%)$ est calculé sur le prix des places de cinéma. Ce taux réduit s'applique également aux abonnements du câble et du satellite ainsi que de la télévision hertzienne à péage. Cette dernière mesure a pour but de faciliter le développement des abonnements et constitue ainsi une aide indirecte au cinéma français, en permettant l'accroissement de la somme qui constitue la base pour le calcul des quotas de production.

Deuxièmement, un régime d'amortissement des investissements film avantageux est offert aux producteurs.

Finalement, l'investissement dans l'industrie cinématographique est stimulé grâce au fonctionnement des sociétés de financement du cinéma et de l'audiovisuel, crées par la loi du 11/7/1985. Ces sociétés anonymes d'investissement sont destinées à la collecte de fonds consacrés exclusivement au financement d'œuvres audiovisuelles et cinématographiques agrées par le CNC. L'investissement dans ces sociétés présente de considérables avantages fiscaux. Ainsi, toute personne physique, ayant souscrit, peut bénéficier d'une déduction des sommes investies dans la limite de $25 \%$ de son revenu imposable et les sociétés actionnaires des soficas d'un amortissement exceptionnel (50\% du montant versé).

L'investissement des soficas dans la production cinématographique en 2002 s'est élevé à 33,42 mln EUR et bien que leur participation dans l'investissement du secteur soit, depuis quelques années, en relative baisse par rapport à d'autres sources de financement, les soficas ont jouée un rôle

21 Des adaptations majeures de la reglemantation concernant le fonctionnement du Compte de soutien ont eu lieu en 1988, 1992 et 1999. 
important dans le développement de l'industrie cinématographique et audiovisuelle en France. De 1985 à 2000, c'est 458 mln d'EUR, qui ont pu être collectés, grâce au fonctionnement de 10 sociétés de ce type, agréées par le Ministère des Finances et le Ministère de la Culture et de la Communication $^{22}$.

\section{d) Le fond de garantie}

Etant donné que l'activité et l'investissement dans la branche cinématographique ont toujours présentes des risques élevés, les pouvoirs publics français ont mis en place un système de garanties financières afin de faciliter l'obtention de crédits par les acteurs de la branche.

Depuis 1983, cette mission a été confiée à un établissement public: l'Institut pour le financement du cinéma et des industries de programmes (IFCIC) dont les fonctions principales sont d'apporter des garanties à des prêts participatifs et des crédits d'investissement, de faciliter le financement des différentes branches du secteur cinématographique par l'apport de sa garantie partielle à des crédits à taux préférentiel ainsi que de distribuer pour le compte de l'Etat les fonds de garantie qui sont dans sa gestion. En 2000, la somme payée dans le cadre de ces fonds de garantie s'élevait à $31,64 \mathrm{mln} \mathrm{EUR.}{ }^{23}$

\section{e) Les autres aides}

A côté des éléments du dispositif de soutien au cinéma français déjà énumérés (obligation imposées aux chaînes de télévision, les aides du Compte de soutien, le soutien fiscal et le fond de garantie placé sous la tutelle de l'Etat), la cinématographie française bénéficie de nombreuses aides aussi bien au niveau national, territorial qu'international, qui illustrent la multiplicité des formes que peut prendre le soutien et confirment la complexité et l'entendue de la politique française dans le domaine culturel.

Au niveau national, l'industrie cinématographique profite de certains fonds gérées par le Ministère de la Culture et de la Communication et des

\footnotetext{
22 R. Bonnel, op. cit., p. 234.

23 Ibidem, p. 591.
} 
aides du Fond d'Action Sociale ${ }^{24}$. La promotion de la cinématographie française est également l'un des éléments forts de la politique culturelle française à l'étranger. En effet, c'est depuis 1984, que le Ministère des Affaires Etrangères mène avec le $\mathrm{CNC}$ une politique de coopération avec d'autres pays, faisant principalement partie de la Zone de Solidarité Prioritaire, dans le domaine de la cinématographie. De nombreuses bourses ${ }^{25}$, prenant surtout la forme d'aides à la production et à la distribution sont octroyés annuellement dans le cadre de cette politique par le Ministère.

En surplus des subventions au niveau national, les cinéastes français bénéficient également de nombreuses aides au niveau régional, départemental ainsi que celui des villes et communautés urbaines.

Mais un rôle croissant pour le développement du cinéma et de la coopération internationale dans ce domaine, jouent les aides internationales parmi lesquelles il faut énumérer les programmes Eurimages et MEDIA (MEDIA, MEDIA II, MEDIA PLUS) de l'Union Européenne.

\section{f) La participation des fonds encadrés dans l'ensemble des investissements dans la production cinématographique}

Les investissements dans la totalité de la production cinématographique française, en 2001, ont été de $905 \mathrm{mln}$ d'EUR, dont 749,12 $\mathrm{mln}$ d'EUR ont été consacrés à la production de films d'initiative française ${ }^{26}$. Les financements encadrés représentent depuis plusieurs années à peu prés la moitié de la totalité de la somme consacrée à la production de films d'initiative française $(382,62 \mathrm{mln}$ d'EUR en 2001). Le tableau ci-joint présente en détails la participation de différents acteurs de la branche cinématographique (chaînes de télévision, soficas, compte de soutien, producteurs, distributeurs etc.) dans le financement de la production française.

${ }^{24}$ Crée en 1984 sous la tutelle du Ministère de l'Emploi et de la Solidarité, le Fond de Solidarité Sociale a pour mission de faciliter l'intégration des immigrants dans la société française.

${ }^{25}$ Fonds Images de France, La Bourse Louis Lumiere, l'Aide au developpement des cinemas du Sud, Fonds Sud Cinema, Fonds Audiovisuel de Coopération Culturelle Internationale au Développement.

${ }^{26}$ Un film d'iniciative française est une production réalisée entièrement ou en sa majeure partie grâce à des apports français. 
La structure de financement des films d'iniciative française

(1987-2002) [en \%]

\begin{tabular}{|l|c|c|c|c|c|c|c|c|c||}
\hline & $\mathbf{1 9 8 7}$ & $\mathbf{1 9 9 3}$ & $\mathbf{1 9 9 6}$ & $\mathbf{1 9 9 7}$ & $\mathbf{1 9 9 8}$ & $\mathbf{1 9 9 9}$ & $\mathbf{2 0 0 0}$ & $\mathbf{2 0 0 1}$ & $\mathbf{2 0 0 2}$ \\
\hline $\begin{array}{l}\text { Financements } \\
\text { encadrés }\end{array}$ & $\mathbf{4 0 , 2}$ & $\mathbf{4 9 , 2}$ & $\mathbf{6 0}$ & $\mathbf{5 3 , 3}$ & $\mathbf{5 5}$ & $\mathbf{5 5 , 8}$ & $\mathbf{5 6 , 1}$ & $\mathbf{5 1 , 3}$ & $\mathbf{4 9 , 9}$ \\
\hline $\begin{array}{l}\text { Aides automati- } \\
\text { ques }\end{array}$ & 4,2 & 7,7 & 8,3 & 7,7 & 7,8 & 6,8 & 6,6 & 9,1 & 7,6 \\
\hline Aides sélectives & 4,5 & 5,5 & 4,9 & 5,2 & 4,4 & 4,4 & 3,6 & 3,2 & 3,4 \\
\hline $\begin{array}{l}\text { Coproductions } \\
\text { des chaînes }\end{array}$ & 7,6 & 5,6 & 10,3 & 7,2 & 7 & 6 & 9 & 3,7 & 4,6 \\
\hline $\begin{array}{l}\text { Préachats } \\
\text { des chaînes }\end{array}$ & 13,4 & 25,2 & 31,7 & 28,7 & 31,5 & 34,2 & 31,2 & 32 & 29,7 \\
\hline \begin{tabular}{l} 
SOFICA \\
\hline $\begin{array}{l}\text { Financements } \\
\text { non-encadrés }\end{array}$
\end{tabular} & $\mathbf{5 9 , 8}$ & $\mathbf{5 0 , 8}$ & $\mathbf{4 0}$ & $\mathbf{4 6 , 7}$ & $\mathbf{4 5}$ & $\mathbf{4 4 , 2}$ & $\mathbf{4 3 , 9}$ & $\mathbf{4 8 , 7}$ & \\
\hline $\begin{array}{l}\text { Producteurs } \\
\text { français }\end{array}$ & 40,3 & 33,4 & 24,3 & 33,4 & 27,9 & 27,9 & 31,9 & 34,6 & 31,5 \\
\hline $\begin{array}{l}\text { A valoir } \\
\text { des distributeurs }\end{array}$ & 14,6 & 5,1 & 5,5 & 3,5 & 6,8 & 8,8 & 5,5 & 6,0 & 7,5 \\
\hline \begin{tabular}{l} 
Apports étrangers \\
\hline (en M EUR)
\end{tabular} & 4,9 & 12,3 & 10,2 & 9,8 & 10,3 & 7,5 & 6,5 & 8,2 & 11 \\
\hline \hline
\end{tabular}

Source: Centre national de la cinématographie.

\section{Conclusion}

Le dispositif de soutien au cinéma est l'un des meilleurs exemples de l'interventionnisme de l'Etat français dans le domaine des médias. Bien sûr, la politique de soutien à cette industrie fragile, n'est pas exclusivement une spécialité française, puisque un dispositif de soutien au secteur existe dans de nombreux pays ou il prend des formes différentes: subventions directes de l'Etat (Danemark, Espagne, Portugal), taxe sur les revenus de la loterie nationale (Grande Bretagne), subventions à l'exportation (Etats Unis), avantages fiscaux, quotas de production et d'émission etc.

La spécificité du système français d'aides au cinéma, est peut être lié au fait que les subventions directes du budget de l'Etat y sont réduites au minimum et que la majeure partie des fonds pour la cinématographie provient d'une redistribution de revenus dans le cadre du secteur audiovisuel (quotas de production, taxe sur les sociétés de télévision, les billets de cinéma et les cassettes vidéo ainsi que DVDs). 
La télévision, le premier concurrent du cinéma, est aujourd'hui également le premier partenaire de la production cinématographique, en finançant par le biais de coproductions, préachats, et de sa contribution au Compte de soutien près de $40 \%$ d'investissements dans la production de films d'initiative française.

Le deuxième élément important du dispositif est le Compte de soutien financier à l'industrie cinématographique et à l'industrie des programmes audiovisuels, crée en 1948, qui finance aujourd'hui pratiquement tous les acteurs de la branche cinématographique. Grâce aux aides automatiques, le Compte contribue à l'équilibre financier du secteur tout en incitant à de nouveaux investissements grâce à un dispositif d'épargne forcé dans la branche alors que les aides sélectives, parmi lesquels il faut surtout mentionner l'avance sur recettes, servent à remplir des objectifs précis dont: la promotion des nouveaux talents, l'aide à la réalisation de longs métrages tournés en langue étrangère, l'aide aux coproductions internationales ainsi qu'aux courts métrages.

Le système est également complété par de nombreux avantages fiscaux (taux de TVA préférentiel, soficas), dont le but est de stimuler l'investissement privé dans le secteur; par un fond de garantie, qui doit faciliter l'accès aux crédits aux acteurs de la branche cinématographique; ainsi que par plusieurs aides distribuées au niveau national, territorial et international.

Cependant, malgré l'existence d'un dispositif d'aides complexe, le financement des investissements du secteur demeure un problème majeur du cinéma français, surtout dans le contexte de la hausse de coûts de production et promotion de films perceptibles depuis plusieurs années. Dans ce contexte, les récents problèmes de Canal Plus, dus à la baisse constante du nombre de ses abonnés et aux problèmes financiers de Vivendi Universal ne font qu'amplifier la nécessité de rechercher de nouvelles sources de financement pour les films français.

De plus, le système de soutien au cinéma français, construit dans le cadre d'une économie nationale fermé, provoque de nombreuses critiques de la part de Bruxelles concernant notamment: la légitimité de certaines aides (aides aux industries techniques distribuées dans le cadre du Compte de soutien) ou la législation concernant les secteurs interdits à la publicité télévisée; et ne peut survivre à l'époque de l'intégration européenne, qu'au prix d'adaptations continuelles. 


\section{Summary}

The policy to support cinematography in France, which is the first film producer in Europe, has had long tradition. The film ,industry” in its present form began after WWII, on the basis of an extensive financing system provided by three laws on supporting movies passed in 1948, 1953 and 1959. In 1946 the development of cinematography was vested with a special state administration body - National Center for Cinematography, supervised by the Ministry of Culture and Communication, which is still in control of this sector. The system of instruments comprised by the French cultural policy aiming both to protect and to promote this means of communication is extremely extensive. On the one hand television market plays a decisive role in the financial system of French cinematography as individual entities are obligated to transfer a part of its revenue for film making, and they are restricted with respect to the type and origin of the programs they broadcast, which indirectly are to increase interest in cinematography. On the other hand, French cinematography takes advantage of an extensive system of aid funds and guarantees, tax exemptions and subsidies at national, regional and EU level.

\section{Streszczenie}

Polityka wspierania kinematografii we Francji, będącej pierwszym producentem filmów w Europie, ma długą tradycję. Fundamentem rozbudowanego systemu finansowania ,przemysłu” filmowego, który w swoim obecnym kształcie zaczął być tworzony po zakończeniu II wojny światowej, stały się trzy ustawy o pomocy dla kina z lat 1948, 1953 i 1959. W 1946 r. pieczę nad kinematografią powierzono specjalnemu organowi administracji państwowej - Narodowemu Centrum Kinematografii, działającemu pod nadzorem Ministerstwa Kultury i Komunikacji, które po dziś dzień sprawuje nadzór nad tym obszarem gospodarki. Zespół narzędzi francuskiej polityki kulturowej, mającej na celu ochronę, jak również wspieranie rozwoju tego środka przekazu jest niezwykle rozbudowany. $Z$ jednej strony, decydujące znaczenie w modelu ekonomicznym francuskiej kinematografii odgrywa rynek telewizyjny, którego poszczególne podmioty mają obowiązek przekazywać część swoich dochodów na produkcję filmową oraz są poddane ograniczeniom co do rodzaju i pochodzenia emitowanych audycji, mającym wpływać pośrednio na wzrost zainteresowania kinem, $\mathrm{z}$ drugiej zaś, kinematografia francuska korzysta z rozbudowanego systemu funduszy pomocowych i gwarancyjnych, ulg podatkowych i subwencji zarówno na szczeblu narodowym, regionalnym, jak i unijnym. 